\title{
Phase behavior of binary mixtures of thick and thin hard rods
}

\author{
René van Roij ${ }^{\mathrm{a}, *}$, Bela Mulder ${ }^{\mathrm{a}}$, Marjolein Dijkstra ${ }^{\mathrm{b}}$ \\ ${ }^{\mathrm{a}}$ FOM-Institute for Atomic and Molecular Physics (AMOLF), Kruislaan 407, 1098 SJ Amsterdam, \\ The Netherlands \\ ${ }^{\mathrm{b}}$ H.H. Wills Physics Laboratory, University of Bristol, Tyndall Avenue, Bristol BS8 1TL, UK
}

Received 24 March 1998; received in revised form 8 September 1998

\begin{abstract}
Using a straightforward extension of the Onsager-theory for hard rods, we consider the thermodynamic stability of the isotropic (I) and nematic $(\mathrm{N})$ phase of binary mixtures of thick and thin hard rods of the same length. We show that such mixtures not only exhibit the expected I-N ordering transition and the previously predicted depletion driven I-I demixing transition, but also a $\mathrm{N}-\mathrm{N}$ demixing transition driven by the orientation entropy of the thinner rods. For various values of the diameter ratio of the two species we present the phase diagrams, which exhibit $\mathrm{I}-\mathrm{N}, \mathrm{I}-\mathrm{I}$ and $\mathrm{N}-\mathrm{N}$ coexistence, $\mathrm{I}-\mathrm{N}-\mathrm{N}$ and $\mathrm{I}-\mathrm{I}-\mathrm{N}$ triple points, and $\mathrm{I}-\mathrm{I}$ and $\mathrm{N}-\mathrm{N}$ critical points. We also present the results of computer simulations of the $\mathrm{I}-\mathrm{I}$ and $\mathrm{I}-\mathrm{N}$ coexistence for diameter ratio 1:10, which are in good agreement with the theory. (c) 1998 Elsevier Science B.V. All rights reserved.
\end{abstract}

PACS: 05.70.Ce; 64.70.Md; 64.75.+g; 82.70.Dd

Keywords: Hard rods; Binary mixture; Phase diagram; Isotropic and nematic phase

\section{Introduction}

This paper deals with phase transitions in binary mixtures of hard rods. Such systems can be regarded as simple models for mixtures of colloidal rods and stiff polymers, or of binary mixtures of two rodlike colloidal species. Here we focus on the spatially homogeneous isotropic (I) and nematic (N) phase, while disregarding positionally ordered phases like smectic, columnar or crystalline phases.

\footnotetext{
* Corresponding author. Present address: H.H. Wills Physics Laboratory, University of Bristol, Tyndall Avenue, Bristol BS8 1TL, UK. Tel.: +44-117-9289000; ext 8935; fax: +44-117-9255624; e-mail: R.VanRoij@bristol.ac.uk.
} 
The best-known phase transition in fluids of rodlike hard particles is the I-N transition, which takes place when the density or the osmotic pressure is increased sufficiently. The mechanism behind this transition is the competition between orientation entropy (favoring I) and center-of-mass entropy (favoring N), as pointed out for monodisperse rod-systems by Onsager in the 1940s [1]. In 1984, it was shown by Lekkerkerker et al. that the same line of reasoning applies to binary mixtures of longer and shorter hard rods [2]. The main difference between the monodisperse and the bidisperse fluid is, in this respect, the considerable fractionation of the coexisting I and $\mathrm{N}$ phase in the latter [2]. It was not until 1993 that Vroege and Lekkerkerker argued that the I-N transition is not the only phase transition in binary hard-rod fluids, but that one should also expect a demixing transition in the nematic phase of binary mixtures of longer and shorter hard rods [3]. This $\mathrm{N}-\mathrm{N}$ demixing transition, which occurs if the length ratio of the two rod species is more extreme than about $1: 3$, was shown to be the result of a competition between the orientation entropy of the shorter rods (favoring demixing) and the entropy of mixing (favoring mixing). The orientation entropy of the shorter rods favors demixing due to the strong alignment that the long rods impose on the short ones in the mixed nematic phase. The results of Ref. [3] are based on approximate Gaussian orientation distribution functions (ODFs), but a recent analysis [4] of the exact high-density ODF confirmed virtually all conclusions of Ref. [3].

In 1995, it was shown by Sear and Jackson that a binary mixture of hard rods can also exhibit a demixing transition in the isotropic phase [7]. These authors considered a binary mixture of equally long hard spherocylinders, one species with a finite and the other species with an infinitesimally small diameter. We argued recently that this I-I demixing transition in binary mixtures of thick and thin hard rods is driven by the depletion effect $[8,9]$, and we estimated that the I-I coexistence is stable with respect to the $\mathrm{I}-\mathrm{N}$ coexistence if the diameter ratio of the two species is more extreme than about $1: 5$ [9]. The theoretical predictions of stable I-I coexistence have been confirmed in a recent Gibbs-ensemble computer simulation study, in which I-I coexistence in a binary mixture of equally elongated hard rods of different diameters was demonstrated [10].

In this paper we consider binary mixtures of equally long hard rods of different diameters within the Onsager theory. Apart from tedious but straightforward numerical analyses of the I-N phase boundaries, we also investigate the stability of the homogeneous nematic phase. We find a $\mathrm{N}-\mathrm{N}$ demixing transition similar to the one found in binary mixtures of longer and shorter hard rods [3,4]. The main extension of this part of the work over that of Ref. [4] is the analysis of the consolute point of the $\mathrm{N}-\mathrm{N}$ coexistence region, i.e. the critical point where the density difference between the co-existing phases vanishes and they can no longer be distinguished. Here we not only use the dominant high-density scaling form of the ODFs, but also the next order correction in an expansion in inverse powers of the density [4,11]. Inclusion of this correction term reveals the existence of such consolute point for relatively small values of the diameter ratio, and explains why this consolute point disappears when the diameter ratio is sufficiently extreme. We note that the existence of this consolute point could neither be inferred from the Gaussian Ansatz used by Vroege and Lekkerkerker 
in Ref. [3], nor from our own asymptotic scaling analysis presented Ref. [4]. Apart from the $\mathrm{I}-\mathrm{N}$ and $\mathrm{N}-\mathrm{N}$ phase boundaries, we also determine the $\mathrm{I}-\mathrm{I}$ demixing binodals. This calculation leads to a slight modification of our earlier estimate of the minimum diameter asymmetry required to observe stable I-I coexistence, as this earlier estimate was only based on I-I spinodals and I-N bifurcations. The resulting phase diagrams for various values of the diameter ratio are thus surprisingly rich, as they involve I-I, I-N and $\mathrm{N}-\mathrm{N}$ coexistence, $\mathrm{I}-\mathrm{I}-\mathrm{N}$ and $\mathrm{I}-\mathrm{N}-\mathrm{N}$ triple points, and $\mathrm{I}-\mathrm{I}$ and $\mathrm{N}-\mathrm{N}$ critical points.

\section{Helmholtz free energy}

\subsection{Onsager functional}

We consider a binary mixture of $N_{\sigma}$ hard rods of species $\sigma=1,2$ with diameters $D_{\sigma}$ and identical lengths $L$ in a macroscopic volume $V$. We restrict attention to long rods, such that $L \gg D_{1}, D_{2}$, and define the ratio $d=D_{2} / D_{1}>1$. The excluded volume of a $\sigma \sigma^{\prime}$ pair of rods is given by $\left(D_{\sigma}+D_{\sigma^{\prime}}\right) L^{2}|\sin \gamma|$, where $\gamma$ is the angle between the long axes of the two rods [12]. We introduce the second virial coefficient of the thinner rods in the isotropic phase as $b=(\pi / 4) L^{2} D_{1}$. The thermodynamic state of the system can be characterized by the convenient and conventional dimensionless number density $c=\left(N_{1}+N_{2}\right) b / V$ and the composition variable $x=N_{2} /\left(N_{1}+N_{2}\right)$, which is the mole fraction of thicker rods. The free energy (in units of $k_{B} T$ per particle) can then be written as a functional of the ODFs $\psi_{\sigma}(\theta)$,

$$
f=\log c+f_{\text {mix }}+f_{\text {or }}+f_{\text {ex }},
$$

where the free energy of mixing $f_{\text {mix }}$, the orientation free energy $f_{\text {or }}$, and the excess term $f_{\text {ex }}$ due to excluded volume interactions are given by

$$
\begin{aligned}
& f_{\text {mix }}=x \log x+(1-x) \log (1-x), \\
& f_{\text {or }}=4 \pi \int_{0}^{\pi / 2} d \theta \sin \theta\left((1-x) \psi_{1}(\theta) \log \psi_{1}(\theta)+x \psi_{2}(\theta) \log \psi_{2}(\theta)\right), \\
& f_{\text {ex }}=32 c \int_{0}^{\pi / 2} d \theta \int_{0}^{\pi / 2} d \theta^{\prime} \sin \theta \sin \theta^{\prime} K\left(\theta, \theta^{\prime}\right) \\
& \quad \times\left((1-x)^{2} \psi_{1}(\theta) \psi_{1}\left(\theta^{\prime}\right)+x(1-x)(1+d) \psi_{1}(\theta) \psi_{2}\left(\theta^{\prime}\right)+x^{2} d \psi_{2}(\theta) \psi_{2}\left(\theta^{\prime}\right)\right) .
\end{aligned}
$$

Here $\theta$ denotes the polar angle of the long axis of a rod with respect to the nematic director. We also defined the conventional azimuthally integrated kernel $K\left(\theta, \theta^{\prime}\right)=$ $\int_{0}^{2 \pi} d \varphi|\sin \gamma|[4,11,12]$, and we used the nematic up-down symmetry $\psi_{\sigma}(\theta)=\psi_{\sigma}(\pi-\theta)$. For given $c$ and $x$, the stationarity conditions of the functional of Eq. (1) read

$$
\begin{aligned}
& \mu_{1}=\log \psi_{1}(\theta)+\frac{8 c}{\pi} \int_{0}^{\pi / 2} d \theta^{\prime} \sin \theta^{\prime} K\left(\theta, \theta^{\prime}\right)\left(2(1-x) \psi_{1}\left(\theta^{\prime}\right)+x(1+d) \psi_{2}\left(\theta^{\prime}\right)\right), \\
& \mu_{2}=\log \psi_{2}(\theta)+\frac{8 c}{\pi} \int_{0}^{\pi / 2} d \theta^{\prime} \sin \theta^{\prime} K\left(\theta, \theta^{\prime}\right)\left((1-x)(1+d) \psi_{1}\left(\theta^{\prime}\right)+2 x d \psi_{2}\left(\theta^{\prime}\right)\right),
\end{aligned}
$$


where $\mu_{\sigma}$ is the Lagrange multiplier that fixes the normalization $4 \pi \int_{0}^{\pi / 2} d \theta \sin \theta \psi_{\sigma}$ $(\theta)=1$.

\subsection{Isotropic phase}

It is easily checked that the isotropic ODF's $\psi_{\sigma, I}=1 /(4 \pi)$ satisfy Eq. (3) for any $c$ and $x$, and lead to the equilibrium free energy of the isotropic phase

$$
f_{I}(x, c)=\log c+f_{\text {mix }}-\log (4 \pi)+\frac{1}{2} c E(x),
$$

where

$$
E(x)=2(1-x)^{2}+2 x(1-x)(1+d)+2 x^{2} d=2(1+(d-1) x)
$$

is the (dimensionless) avarage excluded volume in the isotropic phase expressed in units of $b$. For later reference we also present the (dimensionless) pressure of the isotropic phase as

$$
P_{I}(x, c)=c^{2} \frac{\partial f_{I}(x, c)}{\partial c}=c+\frac{1}{2} c^{2} E(x) .
$$

Analogously, analytic expressions for the chemical potentials of the two rod species in the isotropic phase also follow directly from $f_{I}(x, c)$.

We showed in Ref. [9] that the Helmholtz free energy of (4) gives rise to an I-I spinodal instability at sufficiently high densities for any $d>1$, and estimated that this instability is stable with respect to the $\mathrm{I}-\mathrm{N}$ transition for about $d>5$. In the sequel we improve this estimate using the $\mathrm{I}-\mathrm{I}$ and $\mathrm{I}-\mathrm{N}$ binodals.

\subsection{Nematic phase}

Anisotropic solutions of Eq. (3) are not known analytically, but existing iterative schemes for pure hard-rod fluids $(x=0,1)$ can be straightforwardly modified to yield numerically exact nematic ODFs for any composition $[2,13]$. We used a generalization of the scheme of Herzfeld et al. [13], involving a grid on the $\theta$-interval $[0, \pi / 2]$, to determine the equilibrium ODFs and hence the equilibrium free energy of the nematic phase. From this the pressure and chemical potentials of the nematic phase follow easily, and hence in combination with Eq. (4) the I-N coexistence as a function of $x$ for given diameter ratios.

In principle this well known and fast-converging numerical scheme can also be used to study the possibility of $\mathrm{N}-\mathrm{N}$ coexistence. In practice, however, it turns out that the $\mathrm{N}-\mathrm{N}$ demixing even takes place at such high densities (where the ODFs are extremely peaked about $\theta=0$ ) that even very fine $\theta$-grids become too crude to provide enough numerical resolution. For this reason we study the stability of the nematic phase using the scaling properties of the ODFs, with which we have recently analyzed pure hard-rod systems [11] and binary mixtures of longer and shorter hard rods [4]. Here we go beyond Refs. [4,11] in the sense that we not only calculate the dominant high-density ODF, but also its next order correction, which turns out to be crucial for 
a detailed understanding of the stability of the nematic phase. As we presented the derivation of the dominant contribution in detail in Refs. [4,11], we now mainly focus on the next order correction, and merely sketch the previously obtained results.

The first key ingredient of the scaling approach involves the introduction of the scaled polar angle $t=c \theta$, where $c$ is the dimensionless density. With this definition of $t$ the following high density expansions can be performed

$$
\begin{aligned}
c K\left(\theta, \theta^{\prime}\right) & =K_{0}\left(t, t^{\prime}\right)+K_{2}\left(t, t^{\prime}\right) / c^{2}+\mathcal{O}\left(1 / c^{4}\right), \\
d \theta \sin \theta & =(d t t) / c^{2} \times\left(1-t^{2} / 6 c^{2}+\mathcal{O}\left(1 / c^{4}\right)\right),
\end{aligned}
$$

where the kernels $K_{0}$ and $K_{2}$ are discussed in more detail in Ref. [4]. ${ }^{1}$ The second key ingredient concerns the definition of the so-called reduced ODFs

$$
\phi_{\sigma}(\theta)=\frac{\psi_{\sigma}(\theta)}{\psi_{\sigma}(\theta=0)},
$$

which equal unity at $\theta=0$ by construction. Inserting Eqs. (7) and (8) into the stationarity equations (3) reveals that the reduced ODFs satisfy the expansion

$$
\phi_{\sigma}(\theta)=\phi_{\sigma, 0}(t)+\phi_{\sigma, 2}(t) / c^{2}+\mathcal{O}\left(1 / c^{4}\right),
$$

where the dominant reduced ODFs $\phi_{\sigma, 0}(t)$ and the low density correction $\phi_{\sigma, 2}(t)$ satisfy the following coupled self-consistency equations

$$
\begin{aligned}
\log \phi_{1,0}(t)= & -\left(2 / \pi^{2}\right) \int_{0}^{\infty} d t^{\prime} t^{\prime} \Delta K_{0}\left(t, t^{\prime}\right) \\
& \times\left[2(1-x) \phi_{1,0}\left(t^{\prime}\right) / \Xi_{1}+x(1+d) \phi_{2,0}\left(t^{\prime}\right) / \Xi_{2}\right], \\
\log \phi_{2,0}(t)= & -\left(2 / \pi^{2}\right) \int_{0}^{\infty} d t^{\prime} t^{\prime} \Delta K_{0}\left(t, t^{\prime}\right) \\
& \times\left[(1-x)(1+d) \phi_{1,0}\left(t^{\prime}\right) / \Xi_{1}+2 x d \phi_{2,0}\left(t^{\prime}\right) / \Xi_{2}\right],
\end{aligned}
$$

and

$$
\begin{aligned}
& \phi_{1,2}(t)=-\left(2 / \pi^{2}\right)\left[2(1-x) v_{1}(t)+x(1+d) v_{2}(t)\right] \phi_{1,0}(t), \\
& \phi_{2,2}(t)=-\left(2 / \pi^{2}\right)\left[(1-x)(1+d) v_{1}(t)+2 x d v_{2}(t)\right] \phi_{2,0}(t)
\end{aligned}
$$

with $\Xi_{\sigma}=\int_{0}^{\infty} d t t \phi_{\sigma, 0}(t)$, and $v_{\sigma}(t)$ defined by

$$
\begin{aligned}
v_{\sigma}(t)= & \frac{\int_{0}^{\infty} d t^{\prime} t^{\prime}\left(\Delta K_{0}\left(t, t^{\prime}\right) \phi_{\sigma, 2}\left(t^{\prime}\right)-t^{\prime 2} \Delta K_{0}\left(t, t^{\prime}\right) \phi_{\sigma, 0}\left(t^{\prime}\right) / 6+\Delta K_{2}\left(t, t^{\prime}\right) \phi_{\sigma, 0}\left(t^{\prime}\right)\right)}{\Xi_{\sigma}} \\
& -\frac{\left(\int_{0}^{\infty} d t^{\prime} t^{\prime} \Delta K_{0}\left(t, t^{\prime}\right) \phi_{\sigma, 0}\left(t^{\prime}\right)\right)\left(\int_{0}^{\infty} d t^{\prime} t^{\prime}\left(\phi_{\sigma, 2}\left(t^{\prime}\right)-t^{\prime 2} \phi_{\sigma, 0}\left(t^{\prime}\right) / 6\right)\right)}{\Xi_{\sigma}^{2}} .
\end{aligned}
$$

\footnotetext{
${ }^{1}$ Unfortunately the analytical form of the kernel $K_{2}$ as reported in Ref. [4] is erroneous [5]. The correct expression is given by

$$
K_{2}\left(t, t^{\prime}\right)=\frac{1}{3}\left(t+t^{\prime}\right)\left(\left(t-t^{\prime}\right)^{2} K\left(\frac{4 t t^{\prime}}{\left(t+t^{\prime}\right)^{2}}\right)-3\left(t^{2}+t^{\prime 2}\right) E\left(\frac{4 t t^{\prime}}{\left(t+t^{\prime}\right)^{2}}\right)\right),
$$

where $K$ and $E$ are complete elliptic integrals of the first and second kind, respectively [6]. All numerical results in Ref. [4], however, are unaffected, as they were based on a correct form obtained by using a symbolic manipulation program.
} 
The kernels are defined as $\Delta K_{j}\left(t, t^{\prime}\right)=K_{j}\left(t, t^{\prime}\right)-K_{j}\left(0, t^{\prime}\right)$ for $j=0,2$. Since both Eqs. (10) and (11) are independent of the dimensionless density $c$, they need to be solved for $\phi_{\sigma, 0}$ and $\phi_{\sigma_{2}}$ only once (for a given value of $x$ and $d$ ). The required numerical scheme we used in this calculation is again similar to the one employed by Herzfeld et al. in Ref. [13], but now on a $t$-grid instead of a $\theta$-grid. It turns out that it is sufficient to consider $t \in[0,6]$ only, since for $t>6$ both $\phi_{\sigma, 0}(t)$ and $\phi_{\sigma, 2}(t)$ are vanishingly small for all values of $d$ and $x$. As already pointed out in Ref. [4], one can first determine $\phi_{\sigma, 0}(t)$ for $\sigma=1,2$ from (10), and use the result in Eq. (11) to determine $\phi_{\sigma, 2}(t)$. The resulting ODF at a given $c$ then follows from the normalization condition and the scaling of Eq. (8) as

$$
\psi_{\sigma}(\theta)=\frac{\phi_{\sigma}(\theta)}{4 \pi \int_{0}^{\pi / 2} d \theta \sin \theta \phi_{\sigma}(\theta)}=\frac{c^{2}}{4 \pi \Xi_{\sigma}}\left(\phi_{\sigma, 0}(c \theta)+\frac{1}{c^{2}} \delta \psi_{\sigma, 2}(c \theta)\right),
$$

with $\Xi_{\sigma}$ defined above (12), and with

$$
\delta \psi_{\sigma, 2}(c \theta)=\phi_{\sigma, 2}(c \theta)-\left(\frac{\int_{0}^{\infty} d t t\left(\phi_{\sigma, 2}(t)-t^{2} \phi_{\sigma, 0}(t) / 6\right)}{\Xi_{\sigma}}\right) \phi_{\sigma, 0}(c \theta) .
$$

For a given value of $d$, one can insert the above expression for the equilbrium ODFs into the free energy functional (1), to yield the equilibrium free energy $f_{N}$ (per $k_{B} T$ per particle) of the nematic phase as

$$
f_{N}(x, c)=3 \log c+f_{0}(x)-f_{2}(x) / c^{2}+\mathcal{O}\left(1 / c^{4}\right) .
$$

We do not display the expressions for $f_{0}(x)$ and $f_{2}(x)$ explicitly in terms of the functions $\phi_{\sigma, 0}(t)$ and $\phi_{\sigma, 2}(t)$, since the algebra involved is tedious but straightforward. We merely note that the main features of $f_{N}(x, c)$ are similar to those encountered for mixtures of longer and shorter rods [4], and those of pure rods [11]. More specifically, this implies that (i) the contribution from $f_{\text {ex }}$ to $f_{0}$ is an $x$-independent value +2 , (ii) $f_{\text {or }}$ and $f_{\text {mix }}$ give $x$-dependent contributions to $f_{0}(x)$, (iii) the term $f_{\text {or }}$ also contributes $\log c^{2}=2 \log c$, which together with the ideal gas contribution gives the first term in the right hand side of Eq. (15), (iv) the low-density correction term $-f_{2}(x) / c^{2}$ contains contributions from both $f_{\mathrm{ex}}$ and $f_{\text {or }},(\mathrm{v}) f_{2}(x)$ is positive for all $x$, since the $\mathcal{O}\left(1 / c^{2}\right)$ correction to the ODFs leads to a (slightly) better approximation of the truely minimizing ODFs, and hence to a more negative free energy than the asymptotic high- $c$ ODFs. We conclude from Eq. (15) that the free energy of the nematic phase at any density $c$ is completely well characterized by only two functions, $f_{0}(x)$ and $f_{2}(x)$, which can be determined numerically for given values of the diameter ratio $d$. In Figs. 1 and 2 we plot $f_{0}(x)$ and $f_{2}(x)$, respectively, for several values of $d$. For clarity, we have subtracted a fit linear in $x$ from the numerically determined $f_{0}(x)$ (but not from $f_{2}(x)$ ). This linear contribution is arbitrary and does not influence the phase behavior of the system; it can be absorbed as irrelevant additive constants in the chemical potentials of the two rod-species. 


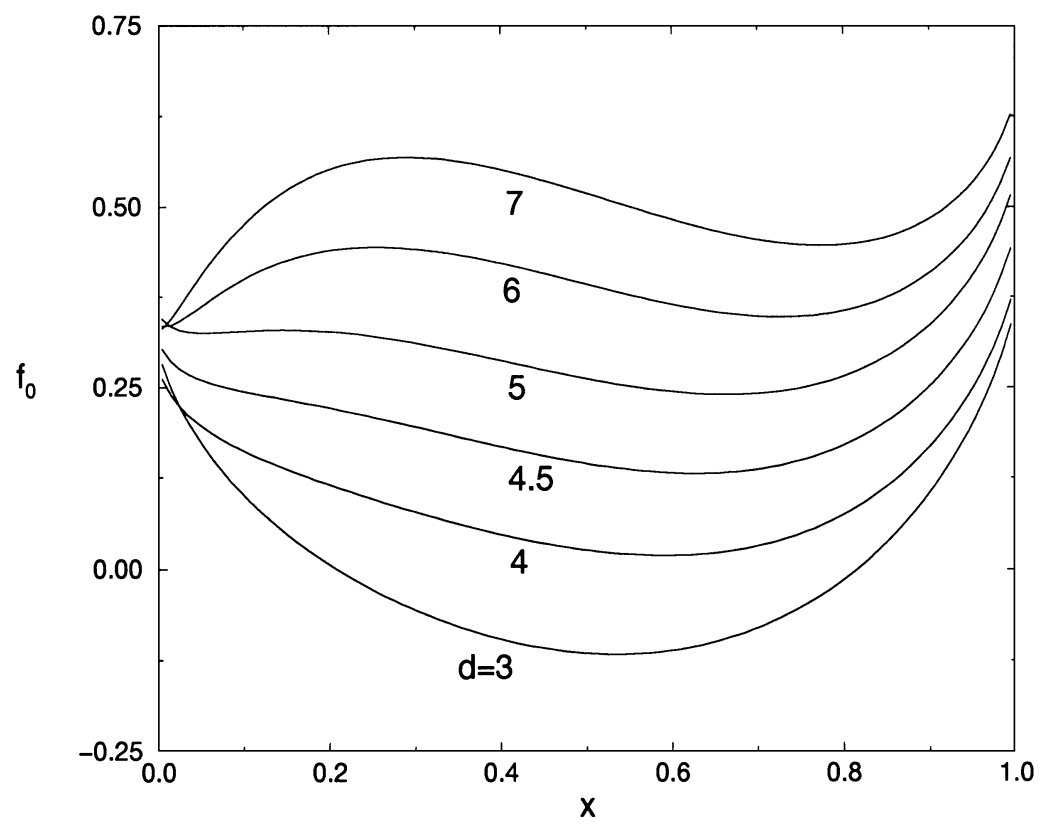

Fig. 1. The asymptotic high-density free energy contribution $f_{0}$, defined in Eq. (15), as a function of the composition $x$ for several values of the diameter ratio $d$. The curvature of $f_{0}$ is positive for all $x$ if $d<d_{c}=4.29$, while there is a composition regime where it is negative if $d>d_{c}$. Note that as explained in the text terms linear in the composition $x$ have been subtracted.

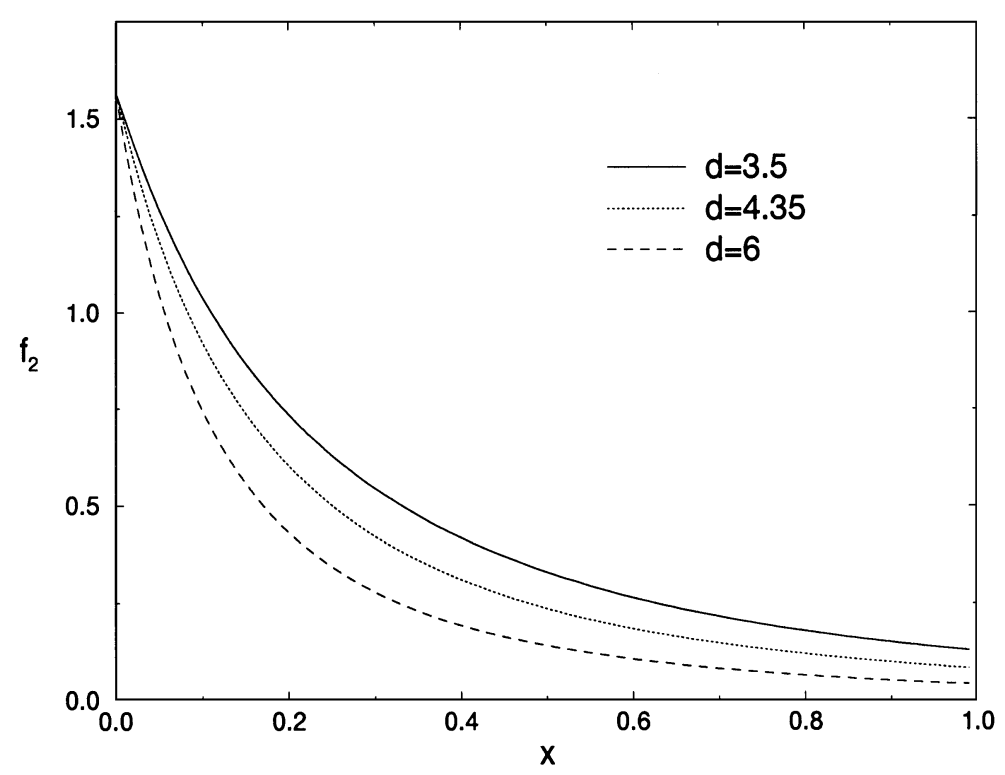

Fig. 2. The $\mathcal{O}\left(1 / c^{2}\right)$ contribution $f_{2}$ to the free energy, given in Eq. (15), as a function of the composition $x$ for several values of the diameter ratio $d$. For all values of $d$ the curvature of $f_{2}$ is positive. 
As we present the phase diagrams - to be discussed in Section 4 - in terms of the pressure, we display the (dimensionless) pressure of the nematic phase explicitly as

$$
P_{N}(x, c)=c^{2} \frac{\partial f_{N}(x, c)}{\partial c}=3 c+2 f_{2}(x) / c .
$$

It is also straightforward to express the chemical potentials in terms of $f_{0}(x)$ and $f_{2}(x)$. We remark that the $1 / c^{2}$-expansions of $f_{N}(x, c)$ and $P_{N}(x, c)$ follow from the truncated virial expansion (in $c$ ) of the free energy functional (1). The completely different character of the two expansions is, of course, caused by the nonlinear self-consistency relations (3), that we transformed into Eqs. (10) and (11).

\section{Stability of the nematic phase}

We showed in Ref. [4] that the isotropic phase, described by $f_{I}(x, c)$ in Eq. (4), exhibits a spinodal demixing instability for any $d>1$. Here we analyse the stability of the nematic phase as a function of $d$. As in Refs. [4,9] we first transform the Helmholtz free energy, here given in Eq. (15) in terms of the composition and the density, into the corresponding Gibbs free energy as a function of composition and pressure. This transformation is facilitated by the analytic inversion of the equation of state (16), which yields the dimensionless density of the nematic phase

$$
c_{N}(x, P)=\frac{P+\sqrt{P^{2}-24 f_{2}(x)}}{6},
$$

as a function of the composition and the (dimensionless) pressure. Clearly, this inversion only makes sense at sufficiently high pressures, where $P^{2}>24 f_{2}(x)$; at lower pressures the present high-density breaks down, and the $1 / c^{2}$ expansion should be carried out to higher order. The Gibbs free energy $g_{N}(x, P)$ of the nematic phase follows directly from the relation $g_{N}(x, P)=f_{N}\left(x, c_{N}(x, P)\right)+P / c_{N}(x, P)$ and Eq. (17), and reads

$$
\begin{aligned}
g_{N}(x, P) & =3 \log c(x, P)+f_{0}(x)-\frac{f_{2}(x)}{c(x, P)^{2}}+\frac{P}{c(x, P)} \\
& \approx 3 \log (P / 3)+f_{0}(x)+3-\frac{9 f_{2}(x)}{P^{2}}+\mathcal{O}\left(1 / P^{4}\right) .
\end{aligned}
$$

If we neglect the $\mathcal{O}\left(1 / P^{4}\right)$ terms in Eq. (18), the thermodynamic stability of the nematic phase is governed by the sign of

$$
\left(\frac{\partial^{2} g_{N}}{\partial x^{2}}\right)_{P}=\frac{d^{2} f_{0}(x)}{d x^{2}}-\frac{9}{P^{2}} \frac{d^{2} f_{2}(x)}{d x^{2}},
$$

i.e. by the sign of the curvature of the Gibbs free energy as a function of the composition at fixed pressure [4]. If this curvature is negative for a given value of $P$ in a particular regime of $x$, then the homogeneous nematic phase is not stable and demixing takes place. If this curvature is positive, the homogeneous nematic phase is stable. The spinodal is defined as the collection of state points at which this curvature vanishes. 
From Eq. (19) it is clear that at sufficiently high pressures the stability is entirely determined by the curvature of $f_{0}(x)$. In Fig. 1 we see that $f_{0}(x)$ is convex at all $x$ if the diameter ratio $d$ is smaller than a critical value $d_{c}$. If $d>d_{c}$, there is a regime of compositions where $f_{0}(x)$ is concave. In this regime the homogeneous nematic phase is unstable. The numerical value of $d_{c}$ can be determined from the condition $C\left(d_{c}\right)=0$, where $C(d)$ is the $d$-dependence of the minimum curvature of $f_{0}(x)$, given by

$$
C=\min _{x \in[0,1]} \frac{d^{2} f_{0}(x)}{d x^{2}} .
$$

This procedure, which we also used in Ref. [4] for mixtures of shorter and longer hard rods, leads in the present case to $d_{c} \approx 4.29$.

Some more insight into the thermodynamics can be obtained by considering the $\mathrm{N}-$ $\mathrm{N}$ demixing spinodal. The spinodal pressure $P_{s}(x)$ is the solution of $\left(\partial^{2} g_{N} / \partial x^{2}\right)_{P_{s}}=0$, which from Eq. (19) is easily seen to be given by

$$
P_{s}(x)=3\left[\left(\frac{d^{2} f_{2}(x)}{d x^{2}}\right) /\left(\frac{d^{2} f_{0}(x)}{d x^{2}}\right)\right]^{1 / 2} .
$$

Clearly, for a given value of $x$, a physical (positive) spinodal pressure only exists if the argument of the root in Eq. (21) is positive, i.e. if the functions $f_{0}(x)$ and $f_{2}(x)$ are both convex or both concave. From Figs. 1 and 2 we see immediately that $f_{2}(x)$ is convex for any $x$ for all displayed values of $d$ (and indeed for all values of $d$ considered). In contrast, $f_{0}(x)$ is only convex in the whole composition interval $0 \leqslant x \leqslant 1$ if $d<d_{c}$; for $d>d_{c}$ there is a finite composition interval in which $f_{0}(x)$ is concave. Consequently, $P_{s}(x)$ is not a continuous function of $x$ if $d>d_{c}$, as it exhibits positive divergences at the two roots of the denominator in Eq. (21). In that case $P_{s}(x)$ does not exist for compositions between these two roots, and the spinodal region is not closed by a critical point. In other words: for $d>d_{c}$ no remixing transition takes place at sufficiently high pressures (or densities), and the $\mathrm{N}-\mathrm{N}$ phase boundary persists up to infinitely high pressures. In contrast, $P_{s}(x)$ is continuous for $d<d_{c}$, and thus exhibits a critical point above which remixing takes place. Whether or not this so-called consolute point for $d<d_{c}$ is stable with respect to the I-N transition can only be inferred from full binodal calculations, to be discussed below.

\section{Phase diagrams}

Based on the analyses above we have calculated the phase diagrams of binary mixtures of thicker and thinner hard rods for various values of the diameter ratio $d$. Phase coexistence was determined by imposing the standard conditions of equal chemical potentials and equal pressure in the two coexisting phases, where pressure and chemical potential follow readily from the equilibrium free energy. Thus the I-I binodals were numerically determined from $f_{I}$ in (4), and the $\mathrm{N}-\mathrm{N}$ binodals from $f_{N}$ in Eq. (15). In principle we could also have determined the I-N coexistence from $f_{I}$ and $f_{N}$. We decided, however, to calculate the full ODFs from Eq. (3) to determine the I-N coexistence, since the high- $c$ expansion up to $\mathcal{O}\left(1 / c^{2}\right)$ terms is not quantitatively correct 
at the relatively low densities near the I-N transition [11]. A drawback of the use of two different methods to calculate phase equilibria is that the $\mathrm{I}-\mathrm{N}-\mathrm{N}$ triple points, i.e. the points where the I-N and $\mathrm{N}-\mathrm{N}$ coexistence curves cross, do not exactly yield the same pressure in the three supposedly coexisting state points. The differences are small, however, and do not affect the interpretation of the phase diagrams. We present all phase diagrams in the composition-pressure plane, so that coexisting state points (with the same pressure) can be distinguished without the need to draw tie-lines. In all plots of Fig. 3, we have scaled the dimensionless pressure $P$ with the diameter ratio $d$. The full curves denote stable phase boundaries, the dotted curves (parts of) metastable phase boundaries, while triangles connected by dashed lines denote (approximate) triple-points.

The smallest diameter ratio we consider here is $d=3$, for which the phase diagram is given in Fig. 3a. The I-N transition is the only one occuring in this system, although there is also a metastable I-I demixing transition at pressures far above the scale of the plot. The homogeneous nematic phase turns out to be stable with respect to demixing whenever nematic solutions to the self-consistency equations for the ODFs exist, and hence there is no $\mathrm{N}-\mathrm{N}$ demixing here. Note that the $\mathrm{I}-\mathrm{N}$ coexistence shows fractionation such that the coexisting isotropic and nematic phases are, respectively, relatively rich in thinner and thicker rods. This is a manifestation of the fact that the thicker rods have a stronger tendency to exhibit orientational order because of their relatively large excluded volume interactions. A similar fractionation effect was also found by Lekkerkerker et al. in binary mixtures of longer and shorter hard rods [2].

The phase diagram for $d=3.5$ is plotted in Fig. 3b. Again the $\mathrm{I}-\mathrm{N}$ transition is the only stable one. However, apart from metastable I-I demixing far beyond the scale of the plot, there is also metastable $\mathrm{N}-\mathrm{N}$ coexistence, as indicated by the dotted curve. As $d<d_{c}$, it should not come as a surprise that the metastable $\mathrm{N}-\mathrm{N}$ coexistence curve is closed by a consolute point. Note that the fractionation effect that accompanies the $\mathrm{I}-\mathrm{N}$ coexistence has become stronger than in Fig. 3a for $d=3$.

The phase diagram for $d=4$ is presented in Fig. 3c. The main difference with that for $d=3.5$ is the increase of the pressure of the consolute point, such that it is stable with respect to the $\mathrm{I}-\mathrm{N}$ coexistence. Thus apart from the $\mathrm{I}-\mathrm{N}$ transition, which tends to exhibit a reentrant feature at $x \approx 0.8$, the system exhibits stable $\mathrm{N}-\mathrm{N}$ coexistence with a critical end point. In fact, there is also an $\mathrm{I}-\mathrm{N}-\mathrm{N}$ triple point at $P d \approx 54$, where an isotropic phase at $x \approx 0$ coexists with two nematic phases.

In Fig. $3 \mathrm{~d}$ we show the phase diagram for $d=4.2$. The most striking feature is a fully developed $\mathrm{N}-\mathrm{N}$ coexistence region enclosed by a critical point (as $d<d_{c}$ ) at a very high pressure. At such high pressures the $1 / c^{2}$-expansion gives excellent results, so that the location of the $\mathrm{N}-\mathrm{N}$ phase boundaries and its critical point is very accurate. We also note that the I-N transition now shows very strong fractionation and a fully developed re-entrant phenomenon.

In Fig. 3e we show the phase diagram for $d=4.3$, so that $d>d_{c}$. As expected from the analysis above, we no longer find a critical $\mathrm{N}-\mathrm{N}$ point: the $\mathrm{N}-\mathrm{N}$ coexistence persists to infinitely high pressures, and at these high pressures the coexisting compositions are 
(a)
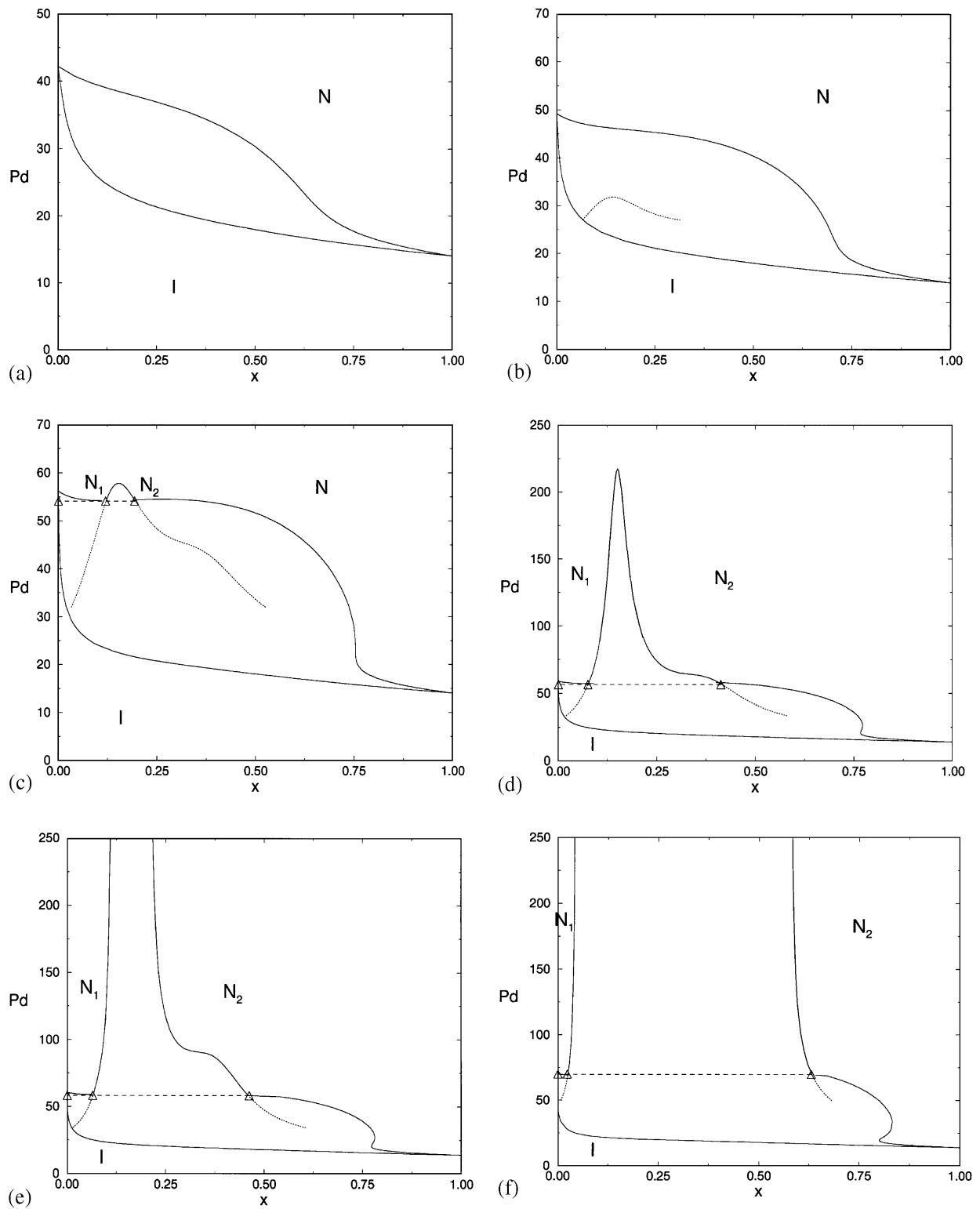

Fig. 3. Phase diagrams in the pressure-composition plane for diameter ratios (a) $d=3$, (b) $d=3.5$, (c) $d=4$, (d) $d=4.2$, (e) $d=4.3$, (f) $d=5$, (g) $d=7$, (h) $d=8$, and (i) $d=10$. The phases are isotropic (I) and nematic $(\mathrm{N})$, and additional labels 1 and 2 are assigned to the cases of isotropic-isotropic or nematic-nematic coexistence. Full curves denote stable phase boundaries, while dotted curves represent (parts of) metastable phase boundaries. Triple points, either I-N-N or I-I-N, are indicated by $\Delta$. Note the presence of a critical (consolute) point on the $\mathrm{N}-\mathrm{N}$ coexistence curves for $3.5 \leqslant d \leqslant d_{c}=4.29$, and its absence for $d>d_{c}$ (see text). Also note that I-I coexistence becomes stable for $d \geqslant 8$. 

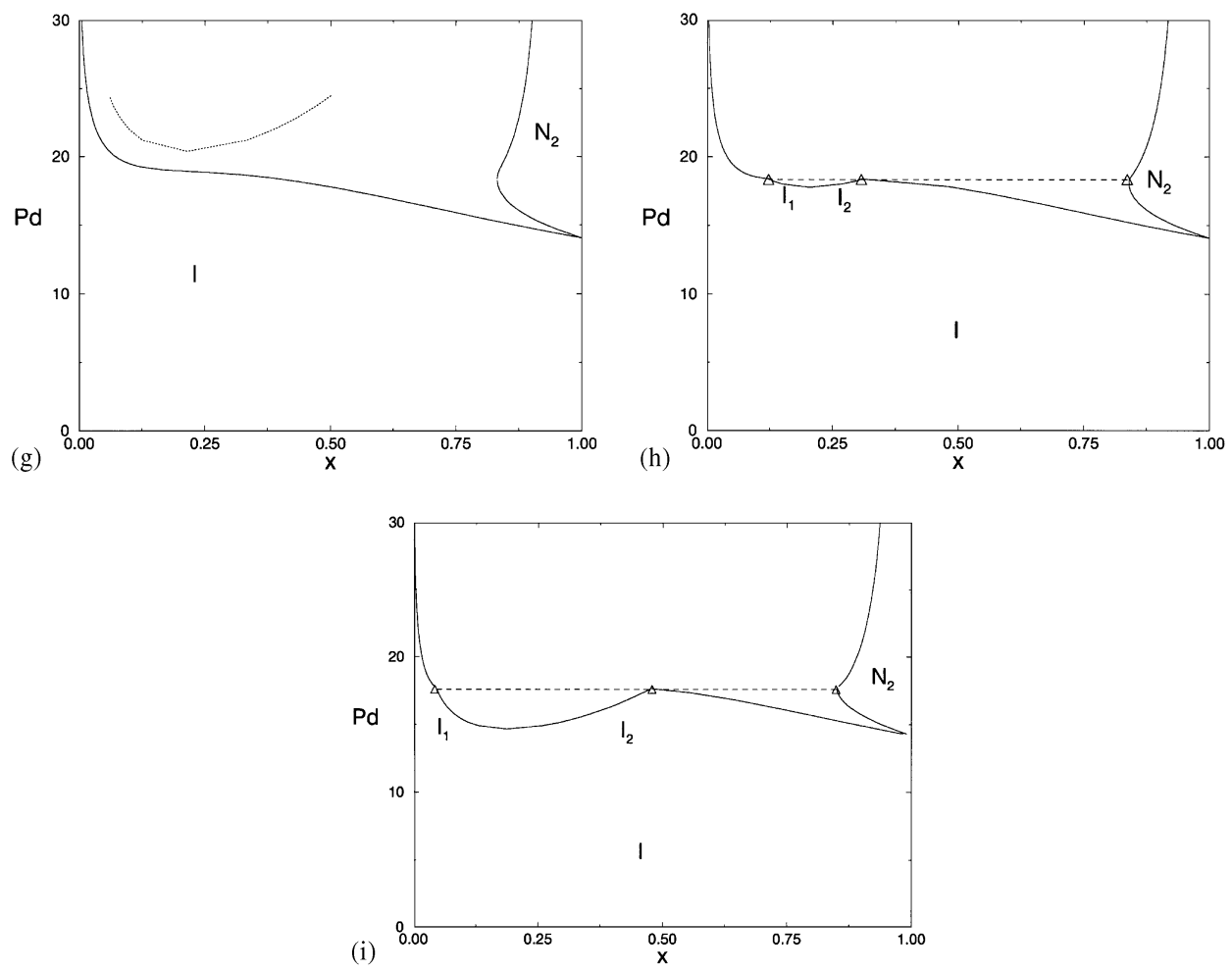

Fig. 3. Continued.

independent of the pressure. A similar phase diagram emerges for $d=5$, shown in Fig. 3f. Here the 'gap of immiscibility' in the nematic phase is even more pronounced, as is the $\mathrm{I}-\mathrm{N}$ fractionation effect.

It was shown in Ref. [9] that one should expect I-I coexistence in binary mixtures of hard rods with different diameters. The reason why we have not encountered I-I coexistence in the phase diagrams of $d \leqslant 5$ is that the corresponding (lower) critical points are far beyond the scale of the plots. In the phase diagram of $d=7$, displayed in Fig. $3 \mathrm{~g}$ in the pressure regime below the very broad N-N coexistence, the (dotted) $\mathrm{I}-\mathrm{I}$ coexistence curve is still metastable with respect to the I-N transition, but it is no longer beyond the scale of the plot. In fact, for $d=8$ Fig. $3 \mathrm{~h}$ reveals that this $\mathrm{I}-\mathrm{I}$ coexistence finally pre-empts the $\mathrm{I}-\mathrm{N}$ coexistence in a small pressure and composition regime. Consequently, there is also a thermodynamically stable $\mathrm{I}-\mathrm{I}-\mathrm{N}$ triple point, indicated by the triangles. In Fig. 3i, we see that for $d=10$ the stable $\mathrm{I}-\mathrm{I}$ coexistence regime is even more pronounced, and thus yields triple points of widely different compositions.

In Fig. 4, we have plotted the phase diagram that results from Gibbs ensemble simulations of the I-I and I-N coexistence of a binary mixture of hard spherocylinders with $L / D_{1}=150$ and $L / D_{2}=15$ (so that $d=10$ ). The filled circles denote the II coexistence, the diamonds the $\mathrm{I}_{2}-\mathrm{N}_{2}$ coexistence, and the filled squares the $\mathrm{I}_{1}-\mathrm{N}_{2}$ 


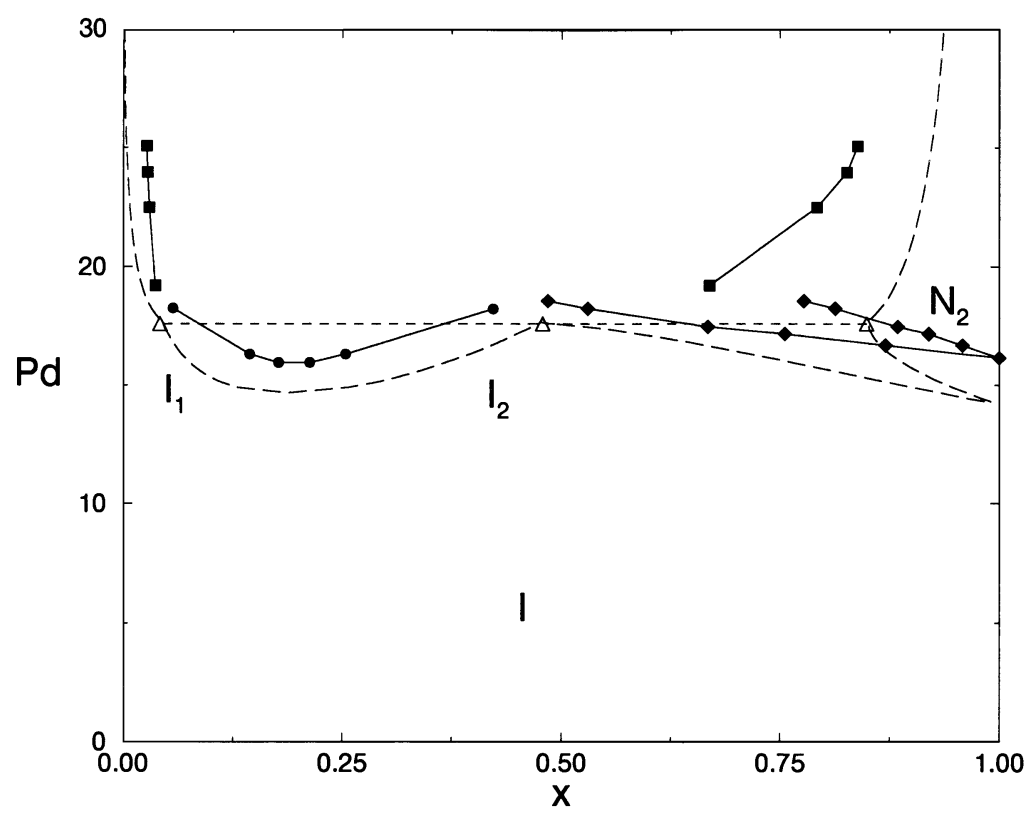

Fig. 4. Comparison of phase diagrams resulting from the Onsager theory (dashed curves) and from Gibbs ensemble simulations (filled symbols and full curves) for a binary mixture of hard rods with diameter ratio $d=10$. The theoretical result is identical to Fig. 3i, and thus holds for infinitely elongated rods. The simulated results stem from Gibbs ensemble simulations of hard spherocylinders with $L / D_{1}=150$ and $L / D_{2}=15$ (so that $\left.D_{2} / D_{1}=d=10\right)$. The black circles represent the simulated $\mathrm{I}_{1}-\mathrm{I}_{2}$ coexistence, and the squares and diamonds the simulated $\mathrm{I}_{1}-\mathrm{N}_{2}$ and $\mathrm{I}_{2}-\mathrm{N}_{2}$ coexistence, respectively. The full curves connecting the simulated state points are a mere guide to the eye, while the open triangles connected by the dashed line represent the theoretical triple points.

coexistence. The full curves connecting the simulated phase boundaries are a mere guide to the eye. For comparison, we have also plotted the theoretical binodals and triple points for $d=10$, represented by the dashed curves and open triangles, respectively. The global agreement between theory and simulation is rather good, although there are quantitative differences. As the thicker rods in the simulations are rather short $\left(L / D_{2}=15\right)$, these (small) differences are most likely to be attributed to the neglect of third- and higher order virial coefficients in the theory, which is justified for infinite, but $n o t$ for finite, aspect ratios of the rods. To give an idea of the microscopic nature of two coexisting isotropic phases, we display in Fig. 5 two typical and equilibrated snapshots that result from the Gibbs ensemble simulations. The first one shows the $\mathrm{I}_{1}$ phase, (with composition $x_{I_{1}}=0.069$ ), and the second one the coexisting $I_{2}$ phase (with composition $\left.x_{I_{2}}=0.411\right)$.

We mention finally that we do not display the high pressure regime, where $\mathrm{N}-\mathrm{N}$ demixing and $\mathrm{I}-\mathrm{N}-\mathrm{N}$ triple points can be found, for $d>5$. We merely state that the trends observed for $d \leqslant 5$ proceed for $d \geqslant 7$, leading to a huge immiscibility gap in the nematic phase. Typically, for $d \geqslant 7$ the $\mathrm{I}-\mathrm{N}-\mathrm{N}$ triple point is such that an isotropic and 


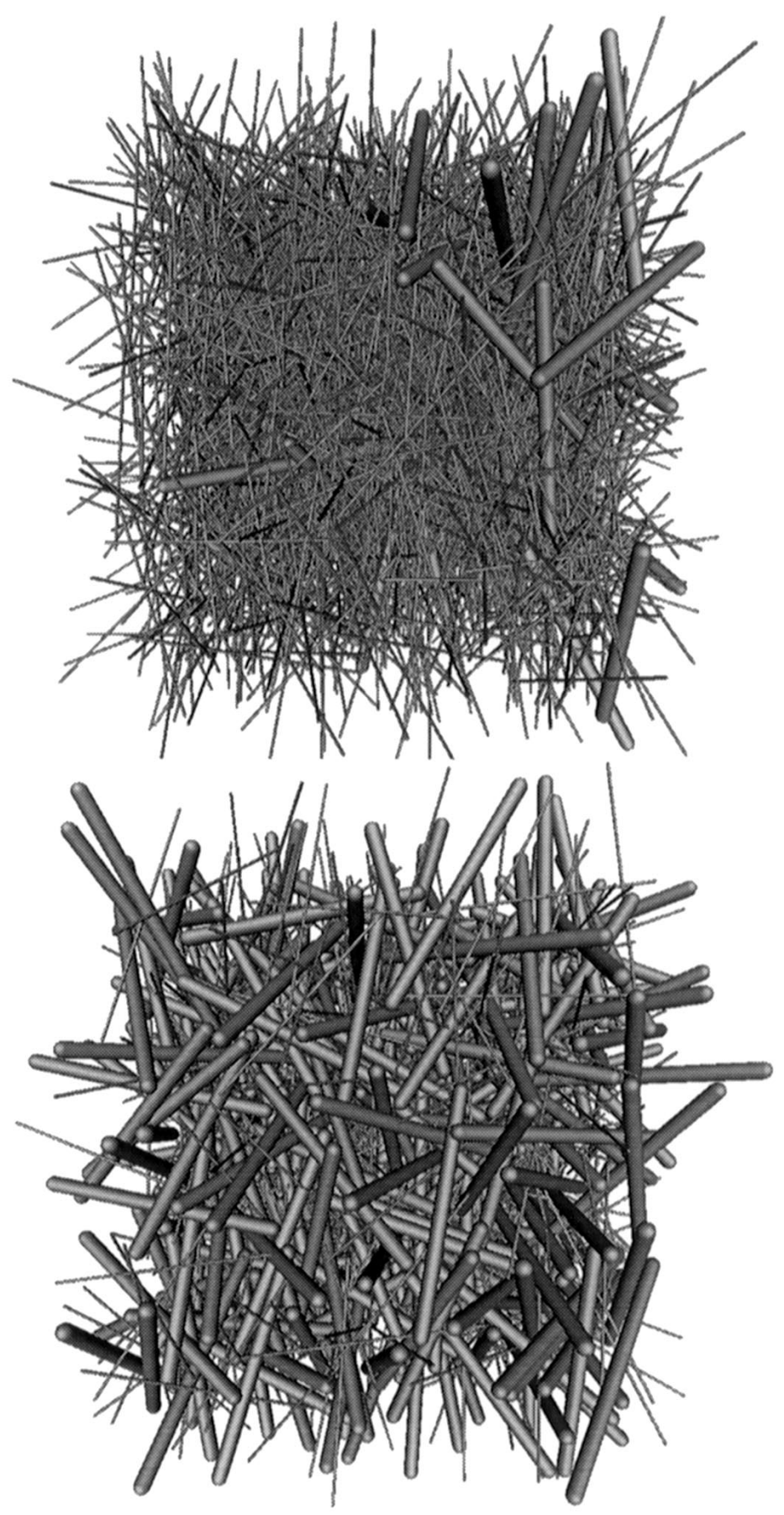

Fig. 5. Snapshots of two coexisting isotropic phases of a binary mixture of hard spherocylinders characterized by length-to-diameter ratios $L / D_{1}=150$ and $L / D_{2}=15$, and hence by a diameter ratio $d=D_{2} / D_{1}=10$. The compositions are $x=0.069$ (upper), and $x=0.411$ (lower). 
nematic phase of virtually pure thinner rods coexist with an almost pure and very well ordered nematic phase of thicker rods.

\section{Summary and discussion}

In this paper we have performed full calculations of the phase diagram of binary mixtures of thicker and thinner hard rods. In several respects we went beyond previous work. First, we have demonstrated for the first time that the homogeneous nematic phase is not always stable with respect to demixing into two coexisting nematic phases. Second, we have shown that the coexistence curve of the two nematic phases exhibits a critical (or consolute) point if the diameter ratio of the rods is less extreme than $1: 4.29$, while such consolute point does not exist for more asymmetric diameter ratios. The accurate analysis of the behavior of the consolute point as a function of the diameter ratio was only possible using the dominant and lowest order correction terms of the scaling technique that we recently developed to calculate high-density orientation distribution functions. We expect that a similar consolute point exists in binary mixtures of longer and shorter hard rods if the length ratio is slightly below 3.2, which is the length ratio above which the spinodal is no longer continuous as a function of the composition. Third, we confirmed our previous prediction of phase separation in the isotropic phase, using full binodals now instead of approximate spinodals and bifurcations. We found that the minimum diameter ratio that is required for a thermodynamically stable coexistence of two isotropic phases is slightly less extreme than $1: 8$, which is slightly more asymmetric than our earlier estimate of $1: 5$. The main reason of this difference is the very strong first order character of the isotropic-nematic transition due to fractionation, which causes spinodals and bifurcations to be only rough estimates of the actual phase boundaries. Fourth, we have compared the I-I and I-N binodals that follow from the Onsager functional for infinitely elongated rods with Gibbs ensemble simulations of rods of finite lengths, and found rather good agreement.

The resulting phase diagrams, displayed in Fig. 3 for various values of the diameter ratio $d>1$, show the following features: (i) for any $d$ there is $\mathrm{I}-\mathrm{N}$ coexistence, with a fractionation effect that becomes more pronounced as $d$ increases; (ii) for $4 \leqslant d<4.29$ there is an additional stable $\mathrm{N}-\mathrm{N}$ coexistence regime that ends in a consolute point, while there is also an I-N-N triple point; (iii) for $4.29<d<8$ as in (ii), except that the $\mathrm{N}-\mathrm{N}$ coexistence is no longer closed by a consolute point; (iv) for $d \geqslant 8$ as in (iii) and in addition a stable $\mathrm{I}-\mathrm{I}$ coexistence that terminates in a critical point at low pressures and a $\mathrm{I}-\mathrm{I}-\mathrm{N}$ triple point at higher pressures.

We mention, finally, that phase diagrams involving $\mathrm{I}-\mathrm{I}, \mathrm{I}-\mathrm{N}$, and $\mathrm{N}-\mathrm{N}$ coexistence have also been found recently in model calculations of thermotropic binary mixtures of side-chain liquid crystalline polymers and low molecular weight liquid crystals [14]. These calculations are, however, based on a combination of Maier-Saupe and FloryHuggins theory, in which the temperature is the driving parameter while the density or pressure is not considered explicitly. Consequently, the mechanisms behind the phase 
transitions discussed in Ref. [14] are energetic rather than entropic in nature, although one could argue that some entropic effects are hidden in model parameters such as the temperature independent part of the Flory-Huggins interaction parameter. It would therefore be interesting to consider a full theory for such binary mixtures, in which both the energetic contributions of Ref. [14] and the entropic contributions discussed in this paper are taken into account explicitly and consistently.

Finally, a few words on the experimental realizability of the type of systems we discuss here. A lower limit on the diameter of the thin particle would be of the order $D_{1} \approx 10 \mathrm{~nm}$ in order for it to be realistic as a stiff colloid (cf. $D_{1}=18 \mathrm{~nm}$ for the Tobacco Mosaic Virus). The first interesting phenomena ( $\mathrm{N}-\mathrm{N}$ demixing) occur for mixtures with a diameter ratio $d \approx 5$, thus requiring a diameter of $D_{2} \approx 50 \mathrm{~nm}$ for the 'thick' particle. In order for the Onsager approximation to be reliable for the 'thick' particle we would need an aspect ratio of at least $x=L / D=10$, implying a length of $L_{2}=500 \mathrm{~nm}$, still comfortably in the colloidal domain. To observe the depletion driven I-I demixing we need diameter ratio's of $d \approx 10$, which would put the required length for the 'thick' particle at a probably unrealistic value of $L_{2}=1000 \mathrm{~nm}$. We thus conclude that the best option would be to look for the $\mathrm{N}-\mathrm{N}$ demixing. The required particle dimensions are at the outer limits of what can be reached using current state of the art techniques for the production of sterically stabilized colloids, e.g., the Boehmite rods produced at the van 't Hoff Laboratory in Utrecht $[15,16]$.

We conclude with the observation that mixtures of long hard rods with different diameters exhibit a wealthy phase behavior in the isotropic and nematic phase. For shorter rods one should also consider inhomogeneous phases like smectic, columnar and crystalline phases, at least at high densities, while in the presence of (anisotropic) attractive interactions the temperature becomes an additional important variable that can drive phase transitions. Clearly, including such effects would lead to even wealthier phase diagrams, the character of which we leave as an open problem.

\section{Acknowledgements}

This work is part of the research programme of the 'Stichting voor Fundamenteel Onderzoek der Materie (FOM)', which is financially supported by the 'Nederlandse organisatie voor Wetenschappelijk Onderzoek (NWO)'. M.D. gratefully acknowledges financial support from the EPSRC grant GR/K 80549.

\section{References}

[1] L. Onsager, Ann. NY Acad. Sci. 51 (1949) 627.

[2] H.N.W. Lekkerkerker, Ph. Coulon, R. van der Haegen, R. Deblieck, J. Chem. Phys. 80 (1984) 3427.

[3] G.J. Vroege, H.N.W. Lekkerkerker, J. Phys. Chem. 97 (1993) 3601.

[4] R. van Roij, B. Mulder, J. Chem. Phys. 105 (1996) 11237.

[5] R. van Roij, B. Mulder, J. Chem. Phys. 109 (1998) 1584. 
[6] We use the conventions as given in: Abramovitz and Stegun, Handbook of Mathematical Functions, 17.3.1-3, Dover, New York, 1972.

[7] R.P. Sear, G. Jackson, J. Chem. Phys. 103 (1995) 8684.

[8] S. Asakura, F. Oosawa, J. Chem. Phys. 22 (1954) 1255; A. Vrij, Pure Appl. Chem. 48 (1976) 471; T. Biben, J.P. Hansen, Phys. Rev. Lett. 66 (1991) 2215; H.N.W. Lekkerkerker, A. Stroobants, Physica A 195 (1993) 387.

[9] R. van Roij, B. Mulder, Phys. Rev. E 54 (1996) 6430.

[10] M. Dijkstra, R. van Roij, Phys. Rev. E 56 (1997) 5594.

[11] R. van Roij, B. Mulder, Europhys. Lett. 34 (1996) 201.

[12] G.J. Vroege, H.N.W. Lekkerkerker, Rep. Progr. Phys. 55 (1992) 1241.

[13] J. Herzfeld, A.E. Berger, J.W. Wingate, Macromolecules 17 (1984) 1718.

[14] H.W. Chiu, Z.L. Zhou, T. Kyu, L.G. Cada, L.C. Chien, Macromolecules 29 (1996) 1051.

[15] M.P.B. van Bruggen, Langmuir 14 (1998) 2245.

[16] H.N.W. Lekkerkerker, Private communication. 